Sains Malaysiana 50(6)(2021): 1531-1541

http://doi.org/10.17576/jsm-2021-5006-03

\title{
Determination of Elements due to Atmospheric Deposition on Barbula indica Moss at Dalat, Vietnam using NAA and TXRF Techniques
}

(Penentuan Unsur Disebabkan Pemendapan Atmosfera pada Lumut Barbula indica di Dalat, Vietnam menerusi Teknik NAA dan TXRF)

\section{Nguyen An Son*, Le Hong Khiem, Nguyen Thi Minh Sang, Doan Phan Thao Tien \& Ho HuU Thang}

\begin{abstract}
In this paper, two multielement analysis techniques, Neutron Activation Analysis (NAA) and Total Reflection X-ray Fluorescence (TXRF), are combined to detect elemental concentrations in Barbula indica moss collected at Dalat, Vietnam. Combining these two techniques has improved the qualitative detection of elements due to atmospheric deposition on moss samples. The concentrations of 40 elements, including $\mathrm{Na}, \mathrm{Mg}, \mathrm{Al}, \mathrm{Si}, \mathrm{P}, \mathrm{S}, \mathrm{Cl}, \mathrm{K}, \mathrm{Ar}, \mathrm{Sc}, \mathrm{Ti}, \mathrm{V}, \mathrm{Cr}, \mathrm{Mn}, \mathrm{Fe}, \mathrm{Co}$, $\mathrm{Ni}, \mathrm{Cu}, \mathrm{Zn}, \mathrm{As}, \mathrm{Se}, \mathrm{Br}, \mathrm{Kr}, \mathrm{Rb}, \mathrm{Y}, \mathrm{Sb}, \mathrm{I}, \mathrm{Cs}, \mathrm{Ba}, \mathrm{La}, \mathrm{Ce}, \mathrm{Sm}, \mathrm{Eu}, \mathrm{Tb}, \mathrm{Dy}, \mathrm{Yb}, \mathrm{Hf}, \mathrm{Ta}, \mathrm{Pb}, \mathrm{Th}$, and $\mathrm{U}$ in the Barbula indica moss samples collected at 19 locations at Dalat have been determined. It is shown that the air in Dalat is suspected of contamination by $\mathrm{Na}, \mathrm{Mg}, \mathrm{Si}, \mathrm{P}, \mathrm{S}, \mathrm{V}, \mathrm{Mn}, \mathrm{Cu}, \mathrm{Se}, \mathrm{Br}$, and $\mathrm{U}$; slightly contaminated by $\mathrm{Mg}, \mathrm{Cl}, \mathrm{K}, \mathrm{Cr}, \mathrm{Ni}, \mathrm{S}$, and $\mathrm{Ni}$; moderately contaminated by $\mathrm{Sc}, \mathrm{Fe}, \mathrm{Co}, \mathrm{Zn}, \mathrm{As}, \mathrm{Kr}, \mathrm{Rb}, \mathrm{Y}, \mathrm{Sb}, \mathrm{Cs}, \mathrm{Ba}, \mathrm{La}, \mathrm{Ce}, \mathrm{Sm}, \mathrm{Eu}, \mathrm{Dy}, \mathrm{Yb}, \mathrm{Ta}, \mathrm{Pb}$, and Th; and seriously contaminated by Tb. Factor analysis has been used to explain the contamination sources of these elements, including $\mathrm{V}, \mathrm{As}, \mathrm{Fe}, \mathrm{Zn}, \mathrm{Se}, \mathrm{Rb}, \mathrm{Sb}, \mathrm{Cs}, \mathrm{Al}, \mathrm{Cu}$, and $\mathrm{Pb}$ in the investigated area. Four factors have been extracted that can explain $86 \%$ of the total variance, and the results suggest that the main sources of atmospheric pollution in Dalat originate from traffic and windblown dust.
\end{abstract}

Keywords: Atmospheric deposition; Barbula indica; Dalat; NAA; TXRF

\section{ABSTRAK}

Dalam kajian ini, dua teknik analisis berbilang-unsur iaitu Analisis Pengaktifan Neutron (NAA) dan Pendaflor Sinar-X Pantulan Keseluruhan (TXRF) telah digabungkan untuk mengesan kepekatan unsur dalam lumut Barbula indica yang diperoleh di Dalat, Vietnam. Penggabungan dua teknik ini telah meningkatkan pengesanan kualitatifunsur kerana terdapat pemendapan atmosfera pada sampel lumut. Kepekatan 40 unsur, iaitu $\mathrm{Na}, \mathrm{Mg}, \mathrm{Al}, \mathrm{Si}, \mathrm{P}, \mathrm{S}, \mathrm{Cl}, \mathrm{K}, \mathrm{Ar}, \mathrm{Sc}, \mathrm{Ti}, \mathrm{V}, \mathrm{Cr}, \mathrm{Mn}$, $\mathrm{Fe}, \mathrm{Co}, \mathrm{Ni}, \mathrm{Cu}, \mathrm{Zn}, \mathrm{As}$, Se, Br, Kr, Rb, Y, Sb, I, Cs, Ba, La, Ce, Sm, Eu, Tb, Dy, Yb, Hf, Ta, Pb, Th dan U dalam sampel lumut Barbula indica yang diperoleh dari 19 lokasi di Dalat telah ditentukan. Data menunjukkan udara di Dalat disyaki tercemar oleh $\mathrm{Na}, \mathrm{Mg}, \mathrm{Si}, \mathrm{P}, \mathrm{S}, \mathrm{V}, \mathrm{Mn}, \mathrm{Cu}$, Se, Br, dan U; sedikit tercemar oleh $\mathrm{Mg}, \mathrm{Cl}, \mathrm{K}, \mathrm{Cr}, \mathrm{Ni}$, S, dan $\mathrm{Ni}$; sederhana tercemar oleh $\mathrm{Sc}, \mathrm{Fe}, \mathrm{Co}, \mathrm{Zn}, \mathrm{As}, \mathrm{Kr}, \mathrm{Rb}, \mathrm{Y}, \mathrm{Sb}, \mathrm{Cs}, \mathrm{Ba}, \mathrm{La}, \mathrm{Ce}, \mathrm{Sm}, \mathrm{Eu}, \mathrm{Dy}, \mathrm{Yb}, \mathrm{Ta}, \mathrm{Pb}$ dan Th; dan tercemar teruk oleh Tb. Analisis faktor telah digunakan untuk menjelaskan sumber pencemaran unsur-unsur ini, termasuk V, As, Fe, Zn, Se, $\mathrm{Rb}, \mathrm{Sb}, \mathrm{Cs}, \mathrm{Al}$, Cu dan Pb di kawasan yang dikaji. Empat faktor telah diekstrak yang dapat menjelaskan $86 \%$ daripada total varians, dan hasilnya menunjukkan bahawa sumber utama pencemaran atmosfera di Dalat berasal daripada lalu lintas dan debu yang ditiup angin.

Kata kunci: Barbula indica; Dalat; NAA; pemendapan atmosfera; TXRF

\section{INTRODUCTION}

Air pollution threatens the health of people everywhere in the world. Air pollution exposes people to fine particles in polluted air. These fine particles penetrate deep into the lungs and cardiovascular system, causing strokes, heart disease, lung cancer, chronic obstructive pulmonary disease, and respiratory infections. Therefore, air quality monitoring has been carried out regularly in many countries. Vietnam is one of the countries with very high air pollution levels that continue to increase. What is the air quality in Vietnam today? It is still a controversial issue in the scientific community, and the government and ordinary citizens need to know the air quality in Vietnam. At present, data on air quality are lacking in many parts of Vietnam.

Around the world, the moss biomonitoring method to study air pollution has been frequently implemented 
because it is cheap and can be easily carried out over a large area. This method provides data on airborne chemical elements needed to assess air quality and is very suitable for developing countries like Vietnam. The application of the moss technique for surveying elemental atmospheric deposition was developed in the late 1960s (Rühling \& Tyler 1970, 1969, 1968). Additional work by Gjengedal and Steinnes (1990) showed that mosses have a strong capacity to retain trace elements, especially metals. Normally, two moss techniques are used: Native species and active biomonitoring. Using native bryophyte moss species in assessing temporal or spatial changes in atmospheric deposition, Sucharová and Suchara (1998) determined the atmospheric loads of 13 elements (A1, $\mathrm{As}, \mathrm{Cd}, \mathrm{Co}, \mathrm{Cr}, \mathrm{Cu}, \mathrm{Fe}, \mathrm{Mo}, \mathrm{Ni}, \mathrm{Pb}, \mathrm{S}, \mathrm{V}$, and $\mathrm{Zn}$ ). Later, mosses were used for active biomonitoring with moss bags. Fernández and Carballeira (2000) used transplanted mosses of the species Scleropodium purum to determine levels of $\mathrm{Co}, \mathrm{Cr}, \mathrm{Cu}, \mathrm{K}, \mathrm{Ni}, \mathrm{Pb}$, Se, and $\mathrm{Zn}$.

The moss biomonitoring method is a low-cost method that might allow Vietnam to collect air quality data to a much greater extent. Recently, the investigation of air pollution using the moss biomonitoring method has been applied in Hanoi and some other big cities (Doan Phan et al. 2019; Khiem et al. 2020; Nguyen et al. 2018). In this report, the results of an investigation of heavy metal air pollution in Dalat using the moss biomonitoring method are presented. Dalat is a popular tourist resort and air quality in Dalat needs to be regularly monitored to help city managers take measures to improve air pollution to the lowest level. The moss biomonitoring method can provide data for this purpose.

The moss species that have been frequently used in Europe are Pleurozium schreber, Hylocomium splendens, Scleropodium purum, and Hypnum cupressiforme
(Frontasyeva \& Harmens 2020; Frontasyeva et al. 2004; Harmens et al. 2010). However, the earlier-mentioned moss species are rarely found in Asia; instead, other mosses are used, such as Hypnum plumaeforme, Taxithelium instratum, Thuidium tamariscellum, and Barbula indica (Abdullah et al. 2011; Doan Phan et al. 2019; Khiem et al. 2020; Lee et al. 2005; Munar et al. 2014). In the present investigation, the samples of Barbula indica moss were collected at different locations in Dalat. Barbula indica moss is widely distributed and easy to collect in the city. The concentrations of 40 chemical elements in the moss samples were determined by two analytical methods, namely, neutron activation analysis and total reflection X-ray fluorescence. The possible sources of heavy metal air pollution have been identified by the application of factor analysis.

\section{MATERIALS AND METHODS}

\section{SAMPLING AREAS}

Dalat, a tourist city located in Lam Dong province of Vietnam, is about $390 \mathrm{~km}^{2}$ in area. Dalat is located at $11.95^{\circ}$ latitude and $108.44^{\circ}$ longitude and sits approximately $1500 \mathrm{~m}$ above sea level on the Lang Biang Plateau in the southern part of the Central Highlands. Dalat is surrounded by lovely mountains and is in the temperate zone because of the effects of altitude and forest cover. Dalat has dry and rainy seasons each year. Normally, the rainy season is from May to October, and the other months are the dry season.

Nineteen moss samples were taken at different sites in and around Dalat (Figure 1) after the end of the rainy season from October 2018 to May 2019. Some moss samples were collected near roads with different levels of traffic, others were collected near farms, and some were taken near fertilizer factories. This study is intended as a

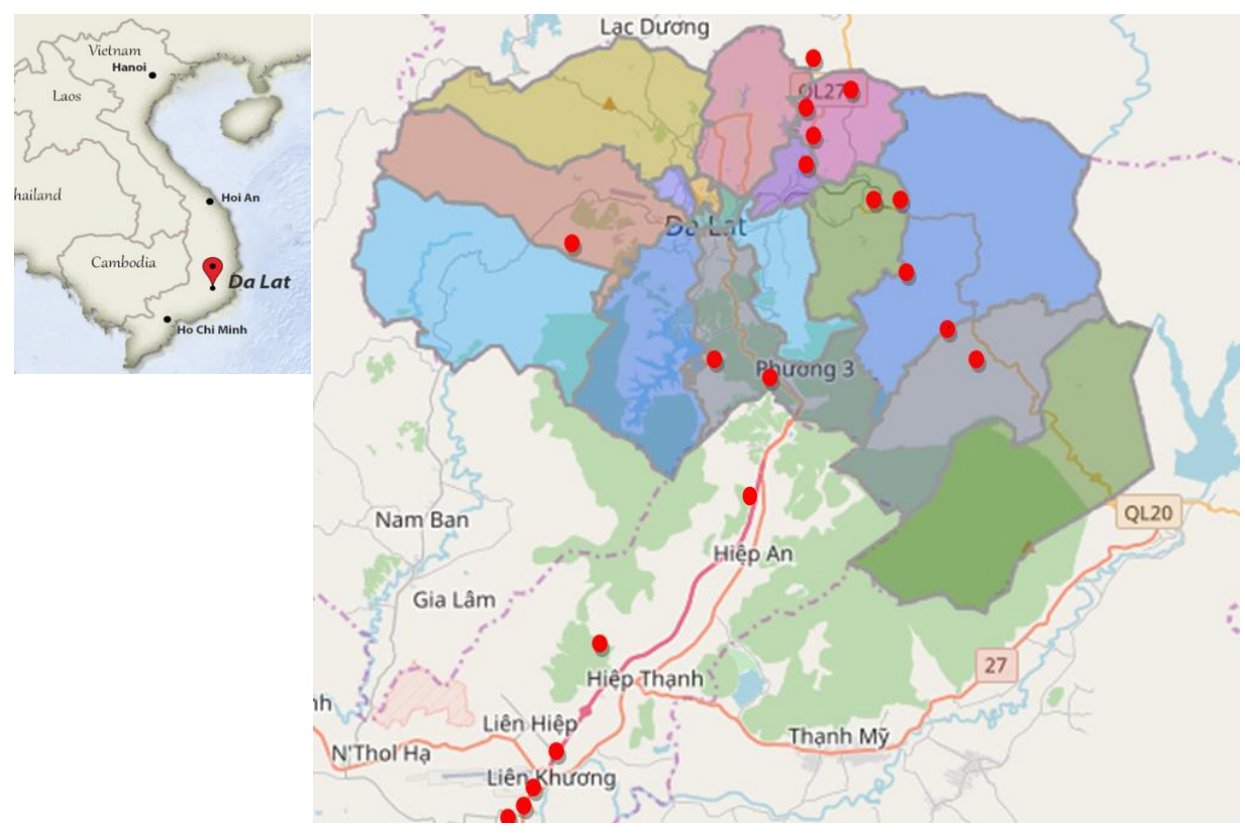

FIGURE 1. Sampling sites in Dalat 

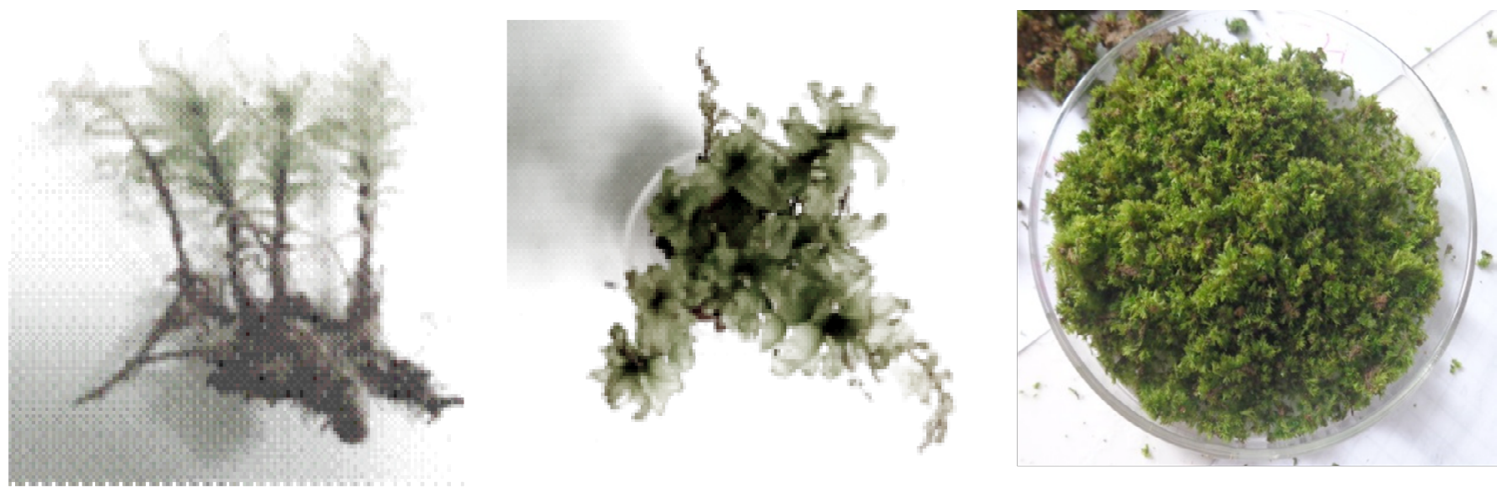

FIGURE 2. Left and Middle: cross section and plan view of Barbula indica moss. Right: a raw moss sample

survey. The main purpose of sampling at a variety of sites is to assess a wide range of possible air pollution sources. A typical moss sample is shown in Figure 2.

\section{PREPARING MOSS SAMPLES}

We followed moss sampling procedures recommended by the ICP Vegetation protocol (Frontasyeva \& Harmens 2020). To minimize the influence of the substrate, moss was collected from trees at least $1.5 \mathrm{~m}$ above the ground and only the green part of the moss was used for analysis. Using only the green portion allows us to measure the relatively recent pollutants from the past two-to-three years. After collection, the moss samples were cleaned of soil particles, washed three times with distilled water, and after that dried at $40{ }^{\circ} \mathrm{C}$ for $50 \mathrm{~h}$. Next, they were crushed and homogenized to a powder $(\sim 0.5 \mathrm{~mm})$ in a
Retsch AS 300 control analytical sieve shaker for $30 \mathrm{~min}$. After preparation, the moss powders were stored in sealed polyethylene vials until they were analyzed.

\section{EXPERIMENTAL TECHNIQUES NAA TECHNIQUE}

NAA was carried out at the $500 \mathrm{~kW}$ Dalat research reactor (DRR) in Vietnam to determine the concentration of trace elements. Previous work established that NAA at DRR meets the requirements of multielement analysis for 42 elements: $\mathrm{Al}, \mathrm{As}, \mathrm{Au}, \mathrm{Ba}, \mathrm{Br}, \mathrm{Ca}, \mathrm{Ce}, \mathrm{Cl}, \mathrm{Co}, \mathrm{Cr}, \mathrm{Cs}, \mathrm{Cu}$, Dy, Eu, Fe, Hf, I, In, K, La, Mg, Mn, Mo, Na, Nd, Pr, Rb, $\mathrm{Sb}, \mathrm{Sc}, \mathrm{Se}, \mathrm{Sm}, \mathrm{Sr}, \mathrm{Ta}, \mathrm{Tb}, \mathrm{Th}, \mathrm{Ti}, \mathrm{U}, \mathrm{V}, \mathrm{W}, \mathrm{Yb}, \mathrm{Zn}$, and $\mathrm{Zr}$ (Ho et al. 2016b).

For NAA sample preparation, the moss powder was heat-sealed in polyethylene foil bags and packed

in aluminum containers. Sample weights were about 50 and $150 \mathrm{mg}$ for short and long-term irradiation, respectively. Table 1 shows the masses of the NAA moss samples.

TABLE 1 . The mass of NAA moss samples in $\mathrm{mg}$

\begin{tabular}{ccc}
\hline Moss sample name & $\begin{array}{c}\text { Mass of moss sample } \\
\text { (short irradiation) }\end{array}$ & $\begin{array}{c}\text { Mass of moss sample (long } \\
\text { irradiation) }\end{array}$ \\
\hline DL01 & 47.25 & 148.51 \\
DL02 & 54.11 & 139.27 \\
DL03 & 57.0 & 189.79 \\
DL04 & 47.0 & 150.57 \\
DL05 & 72.78 & 159.99 \\
DL06 & 55.7 & 151.53 \\
DL07 & 43.91 & 152.57 \\
DL08 & 60.91 & 181.25 \\
DL09 & 65.62 & 156.71 \\
DL10 & 62.47 & 157.05 \\
DL11 & 46.92 & 121.77 \\
DL12 & 52.27 & 171.85 \\
DL13 & 67.05 & 173.39 \\
DL14 & 54.37 & 164.07 \\
DL15 & 55.16 & 122.68 \\
DL16 & 66.25 & 157.81
\end{tabular}




$\begin{array}{cc}\text { DL17 } & 54.34 \\ \text { DL18 } & 50.33 \\ \text { DL19 } & 48.6\end{array}$

The k0-NAA method was used to analyze the moss samples. This method was developed at the Dalat Nuclear Research Institute and has been officially applied since 2002 as the standard method (Ho \& Pham 2003; Ho et al. 2016a).

Short irradiations were conducted for $45 \mathrm{~s}$ on Channel 7-1 at a thermal neutron flux of approximately $4.2 \times 10^{12} \mathrm{~cm}^{-2} \mathrm{~s}^{-1}$. To determine short-lived isotopes, every sample was decayed for 1-2 min and then measured for $15 \mathrm{~min}$. Long irradiations were conducted for $1 \mathrm{~h}$ on a rotary rack at a thermal neutron flux of approximately $3.5 \times 10^{12} \mathrm{~cm}^{-2} \mathrm{~s}^{-1}$. The samples were allowed to decay for 3 days prior to counting for $1 \mathrm{~h}$. The moss samples were measured with a gamma-ray spectrometer on a Canberra HPGe detector, for which the FWHM is approximately 1.9 $\mathrm{keV}$ at $1332 \mathrm{keV}$ and the relative efficiency is $\sim 33 \%$. Genie-2K software was used to analyze the obtained gamma spectra and the K0-Dalat software (Ho et al. 2016a) was used to determine the elemental concentrations from the obtained count rates. Certified international reference materials (SRM-1572, SRM 1547, IAEA-V-10, and SMELS Type I, Type II, and Type III) were analyzed for analytical quality control. The differences between the certified values and those obtained for all analyzed elements are smaller than $7 \%$.
Along with 165.68 we used TXRF to analyze the trace element concentrations in the moss samples. A Bruker S2 PICOFOX spectrometer was used for the TXRF measurements. PRe spectrometer can detect and measure K-line energy of the following elements: $\mathrm{Al}, \mathrm{Si}, \mathrm{P}, \mathrm{Se}, \mathrm{Cl}$, $\mathrm{K}, \mathrm{Ca}, \mathrm{Sc}, \mathrm{Ti}, \mathrm{V}, \mathrm{Cr}, \mathrm{Mn}, \mathrm{Fe}, \mathrm{Co}, \mathrm{Ni}, \mathrm{Cu}, \mathrm{Zn}, \mathrm{Ga}, \mathrm{Ge}, \mathrm{As}$, $\mathrm{Se}, \mathrm{Br}, \mathrm{Rb}, \mathrm{Sr}, \mathrm{Y}$, Es, Fm, Md, No, and Lr. With L-line energy, it can measure elements: $\mathrm{Ru}, \mathrm{Rh}, \mathrm{Pd}, \mathrm{Ag}, \mathrm{Cd}, \mathrm{In}$, $\mathrm{Sn}, \mathrm{Sb}, \mathrm{Te}, \mathrm{I}, \mathrm{Cs}, \mathrm{Ba}, \mathrm{Hf}$, Ta, W, Re, Os, Ir, Pt, Au, Hg, Tl, $\mathrm{Pb}, \mathrm{Bi}, \mathrm{Po}, \mathrm{At}, \mathrm{Fr}, \mathrm{Ra}, \mathrm{La}, \mathrm{Ce}, \mathrm{Pr}, \mathrm{Nd}, \mathrm{Pm}, \mathrm{Sm}, \mathrm{Eu}, \mathrm{Gd}$, Tb, Dy, Ho, Er, Tm, Yb, Lu, Ac, Th, Pa, and U. Difficult or impossible to measure are elements: $\mathrm{Na}, \mathrm{Mg}, \mathrm{Ar}, \mathrm{Kr}$, $\mathrm{Xe}, \mathrm{Rn}, \mathrm{Np}, \mathrm{Pu}, \mathrm{Am}, \mathrm{Cm}$, Ek, Cf , H, He, Li, Be, B, C, N, O, F, Ne, Zr, Nb, Mo, and Tc (Towett et al. 2013). To prepare the TXRF moss samples, the moss powder samples described above were ground down to a grain size of 50 $\mu \mathrm{m}$ using a Retsch MM 400 mixer mill. Then, the moss powder samples must be liquified by means of digestion. In this investigation, MARS 6 Microwave Acid Digestion made by CEM Corporation was used. A $0.5 \mathrm{~g}$ weight of moss powder was placed into the digestion vessel and 10 $\mathrm{mL}$ of $\mathrm{HNO}_{3}(65 \%)$ were added. The mixture was gently swirled and allowed to sit for approximately $15 \mathrm{~min}$ before closing the vessel. The operating time of the Retsch MM 400 mixer mill was $50 \mathrm{~min}$. After finishing this procedure, the moss samples were liquid (the original sample). Then $500 \mu \mathrm{L}$ of the original sample we retransferred to a polymer container, and a gallium internal standard liquid was added until the sample reached $1 \mathrm{ppm}$ gallium. The sample was then thoroughly homogenized with an automatic sample shaker. After thorough homogenization, $10 \mu \mathrm{L}$ of the

TXRF TECHNIQUE

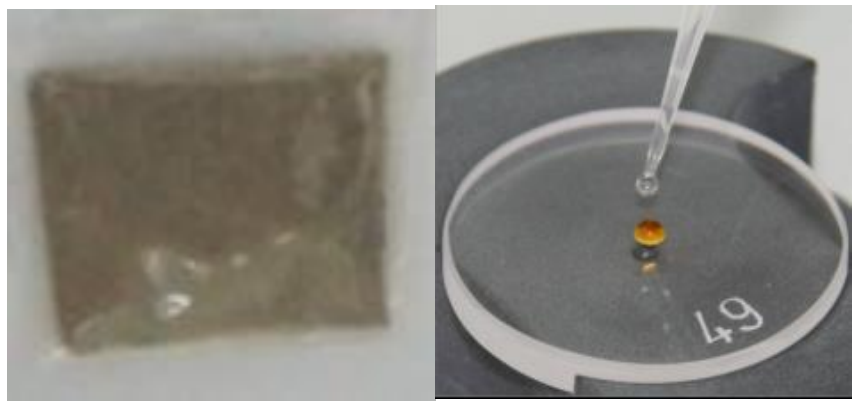

FIGURE 3. Left: NAA sample. Right: TXRF sample

sample were transferred to a sample carrier and then dried at $30{ }^{\circ} \mathrm{C}$. Figure 3 shows an NAA sample and a TXRF sample.

\section{RESULTS AND DISCUSSION}

ORIGINAL DATA
The elemental concentration data of the moss samples from the 19 sampling sites were analyzed using NAA and TXRF techniques. In all, 40 elements were detected, including $\mathrm{Na}, \mathrm{Mg}, \mathrm{Al}, \mathrm{Si}, \mathrm{P}, \mathrm{S}, \mathrm{Cl}, \mathrm{K}, \mathrm{Ar}, \mathrm{Sc}, \mathrm{Ti}, \mathrm{V}, \mathrm{Cr}$, $\mathrm{Mn}, \mathrm{Fe}, \mathrm{Co}, \mathrm{Ni}, \mathrm{Cu}, \mathrm{Zn}, \mathrm{As}, \mathrm{Se}, \mathrm{Br}, \mathrm{Kr}, \mathrm{Rb}, \mathrm{Y}, \mathrm{Sb}, \mathrm{I}, \mathrm{Cs}$, $\mathrm{Ba}, \mathrm{La}, \mathrm{Ce}, \mathrm{Sm}, \mathrm{Eu}, \mathrm{Tb}, \mathrm{Dy}, \mathrm{Yb}, \mathrm{Hf}, \mathrm{Ta}, \mathrm{Pb}, \mathrm{Th}$, and U. 
The error in the concentrations for all elements is less than $10 \%$. The experimental data of Barbula indica moss at Dalat are presented in Table 2. These results show

Chi Minh City using INAA. that more elements were found than in similar studies in Vietnam. Khiem et al. (2020) detected 22 elements in moss samples from Hanoi using the Proton Induced X-ray Emission (PIXE) technique. Doan Phan et al. (2019) found 30 elements in moss samples from Hue, Hoi An, and Ho

TABLE 2. Trace element concentrations for moss samples from Dalat region (in mg. $\mathrm{kg}^{-1}$ )

\begin{tabular}{|c|c|c|c|c|c|c|c|c|c|c|c|c|c|c|c|c|c|c|c|}
\hline \multirow{2}{*}{ Element } & \multicolumn{19}{|c|}{ Site } \\
\hline & DL01 & DL02 & DL03 & DL04 & DL05 & DL06 & DL07 & DL08 & DL09 & DL10 & DL11 & DL12 & DL13 & DL14 & DL15 & DL16 & DL17 & DL18 & DL19 \\
\hline $\mathrm{Na}^{*}$ & 287 & 133 & 389 & 266 & 139 & 138 & 152 & 96 & 183 & 150 & 89 & 216 & 139 & 218 & 234 & 394 & 112 & 150 & 319 \\
\hline Mg* & 236 & 233 & 239 & 252 & 157 & 186 & 179 & 182 & 171 & 170 & 292 & 213 & 290 & 415 & 153 & 339 & 188 & 163 & 218 \\
\hline $\mathrm{Al}^{* *}$ & 2087 & 2125 & 4581 & 2015 & 1639 & 2171 & 3169 & 861 & 5209 & 1885 & 1197 & 2320 & 1636 & 334 & 2865 & 3342 & 1437 & 3122 & 3506 \\
\hline $\mathrm{Si}^{* *}$ & 3210 & 5210 & 11330 & 5341 & 4202 & 5339 & 4524 & 3675 & 3755 & 9386 & 5425 & 2872 & 2975 & 2678 & 2826 & 2875 & 3246 & 2846 & 2759 \\
\hline $\mathrm{P}^{* *}$ & 326 & 1048 & 839 & 591 & 603 & 517 & 1210 & 1111 & 1423 & 851 & 545 & 803 & 958 & 809 & 759 & 1106 & 1049 & 565 & 849 \\
\hline$S^{* *}$ & 548 & 1382 & 1461 & 1018 & 1239 & 703 & 1056 & 1401 & 1493 & 1223 & 1011 & 1211 & 1197 & 1481 & 1124 & 1428 & 1326 & 970 & 1227 \\
\hline $\mathrm{Cl}^{*}, * *$ & 345 & 752 & 297 & 704 & 219 & 781 & 238 & 300 & 216 & 343 & 407 & 924 & 352 & 52 & 1288 & 90 & 1042 & 226 & 343 \\
\hline $\mathrm{K}^{*, * *}$ & 7522 & 2926 & 6208 & 10670 & 4716 & 2526 & 7985 & 5792 & 10940 & 6047 & 2036 & 7099 & 1858 & 4491 & 4729 & 8856 & 8295 & 5427 & 4584 \\
\hline $\mathrm{Sc}^{*}$ & 2.36 & 0.97 & 1.50 & 2.10 & 0.86 & 0.44 & 1.25 & 0.46 & 1.90 & 0.34 & 0.15 & 1.20 & 0.70 & 0.08 & 0.73 & 1.83 & 0.88 & 0.35 & 0.58 \\
\hline $\mathrm{Ti}^{* *}$ & 122 & 139 & 220 & 234 & 119 & 160 & 279 & 95 & 107 & 181 & 84 & 87 & 240 & 28 & 28 & 68 & 44 & 87 & 75 \\
\hline $\mathrm{V}^{*}, * *$ & 7.80 & 9.60 & 7.60 & 12.30 & 5.60 & 9.10 & 7.80 & 5.20 & 9.50 & 12.40 & 5.30 & 8.00 & 9.40 & 5.00 & 17.60 & 16.80 & 10.40 & 5.00 & 18.70 \\
\hline $\mathrm{Cr}^{*},{ }^{* *}$ & 5.90 & 6.10 & 11.80 & 12.70 & 5.80 & 2.98 & 5.69 & 3.44 & 12.20 & 3.86 & 1.35 & 11.10 & 4.50 & 9.11 & 8.25 & 6.90 & 12.86 & 3.12 & 7.97 \\
\hline $\mathrm{Mn}^{*}, * *$ & 138 & 69 & 101 & 58 & 60 & 56 & 50 & 38 & 60 & 47 & 58 & 64 & 77 & 40 & 122 & 84 & 79 & 71 & 163 \\
\hline $\mathrm{Fe}^{*}, * *$ & 4494 & 2727 & 5064 & 2915 & 2191 & 948 & 3244 & 1178 & 5065 & 1018 & 400 & 3088 & 1963 & 286 & 2377 & 2795 & 2709 & 1222 & 2066 \\
\hline $\mathrm{Co}^{*}, * *$ & 1.58 & 0.80 & 1.41 & 0.65 & 0.66 & 0.28 & 1.00 & 0.77 & 0.80 & 0.39 & 0.21 & 1.05 & 1.57 & 0.30 & 1.81 & 1.45 & 2.19 & 0.97 & 1.87 \\
\hline $\mathrm{Ni}^{* * *}$ & 1.08 & 1.46 & 3.02 & 2.14 & 0.86 & 0.86 & 1.28 & 0.68 & 2.56 & 1.12 & 1.15 & 1.06 & 1.10 & 2.50 & 6.10 & 2.66 & 5.58 & 5.63 & 5.90 \\
\hline $\mathrm{Cu}^{* *}$ & 12.34 & 11.94 & 16.28 & 6.76 & 10.78 & 7.50 & 10.60 & 16.80 & 14.06 & 8.74 & 7.12 & 15.80 & 8.20 & 9.21 & 12.50 & 11.60 & 17.86 & 13.45 & 16.66 \\
\hline $\mathrm{Zn} *$,** & 86 & 214 & 176 & 59 & 157 & 80 & 110 & 1366 & 140 & 83 & 110 & 479 & 157 & 47 & 76 & 220 & 802 & 97 & 191 \\
\hline $\mathrm{As}^{*}$ & 11.36 & 2.56 & 5.30 & 1.61 & 1.28 & 2.78 & 4.25 & 1.49 & 6.47 & 3.97 & 0.78 & 7.06 & 2.56 & 0.55 & 0.70 & 0.71 & 0.49 & 0.77 & 0.93 \\
\hline $\mathrm{Se}^{*}$ & 0.34 & 0.16 & 0.21 & 0.25 & 0.16 & 0.09 & 0.21 & 0.11 & 0.24 & 0.19 & 0.10 & 0.15 & 0.15 & 0.10 & 0.21 & 0.25 & 0.10 & 0.11 & 0.20 \\
\hline $\mathrm{Br}^{*}, * *$ & 3.40 & 2.33 & 6.88 & 2.58 & 2.62 & 3.05 & 1.97 & 3.64 & 4.51 & 6.44 & 2.48 & 3.79 & 4.53 & 2.78 & 2.96 & 1.94 & 2.15 & 1.62 & 5.80 \\
\hline $\mathrm{Kr} * *$ & 1.16 & 0.16 & 0.87 & 1.54 & 1.21 & 1.78 & 0.52 & 1.33 & 1.16 & 0.22 & 1.54 & 0.22 & 1.26 & 1.42 & 1.57 & 1.64 & 1.87 & 1.59 & 1.33 \\
\hline $\mathrm{Rb}^{*}, * *$ & 27.40 & 7.80 & 17.80 & 19.20 & 13.90 & 5.21 & 22.20 & 22.00 & 25.30 & 11.10 & 2.95 & 29.00 & 4.70 & 5.47 & 3.10 & 2.70 & 23.00 & 2.96 & 9.20 \\
\hline $\mathrm{Y}^{* *}$ & 28.20 & 1.28 & 1.96 & 7.88 & 0.78 & 1.30 & 1.46 & 1.56 & 4.28 & 1.10 & 1.36 & 1.46 & 1.30 & 0.42 & 3.06 & 7.30 & 2.66 & 6.30 & 6.28 \\
\hline $\mathrm{Sb}^{*}$ & 0.37 & 0.08 & 0.43 & 0.05 & 0.16 & 0.17 & 0.14 & 0.44 & 0.08 & 0.12 & 0.36 & 0.17 & 0.28 & 0.24 & 0.11 & 0.64 & 0.30 & 0.05 & 0.14 \\
\hline I* & 5.90 & 4.10 & 10.60 & 6.00 & 3.20 & 6.02 & 3.10 & 2.70 & 2.70 & 4.74 & 5.22 & 6.70 & 7.10 & 4.80 & 4.50 & 4.74 & 3.70 & 2.10 & 1.24 \\
\hline $\mathrm{Cs}^{*}$ & 1.76 & 0.38 & 0.84 & 1.64 & 0.44 & 0.22 & 0.82 & 0.42 & 1.40 & 0.45 & 0.21 & 0.77 & 0.30 & 0.13 & 0.31 & 0.86 & 0.34 & 0.18 & 0.29 \\
\hline $\mathrm{Ba}^{* *}$ & 2.52 & 26.58 & 22.78 & 3.60 & 24.36 & 18.96 & 12.56 & 8.88 & 14.54 & 4.20 & 7.64 & 7.54 & 5.48 & 3.28 & 4.68 & 12.45 & 5.78 & 20.65 & 15.45 \\
\hline $\mathrm{La}^{*}$ & 23.31 & 2.32 & 3.29 & 23.96 & 1.90 & 3.90 & 2.40 & 1.25 & 2.80 & 1.74 & 1.83 & 3.64 & 1.79 & 0.36 & 3.20 & 6.61 & 1.70 & 18.40 & 4.41 \\
\hline $\mathrm{Ce}^{*}$ & 45.60 & 5.60 & 7.50 & 25.17 & 4.60 & 9.80 & 5.75 & 3.52 & 7.00 & 2.20 & 2.06 & 9.70 & 5.00 & 0.38 & 3.37 & 9.00 & 3.20 & 13.74 & 4.48 \\
\hline Sm* & 5.52 & 0.35 & 0.67 & 3.00 & 0.36 & 0.54 & 0.37 & 0.31 & 0.37 & 0.31 & 0.40 & 0.75 & 0.32 & 0.05 & 0.68 & 1.02 & 0.36 & 2.75 & 0.73 \\
\hline $\mathrm{Eu}^{*}$ & 0.74 & 0.09 & 0.09 & 0.29 & 0.06 & 0.05 & 0.09 & 0.05 & 0.09 & 0.03 & 0.02 & 0.09 & 0.07 & 0.01 & 0.11 & 0.20 & 0.14 & 0.27 & 0.10 \\
\hline $\mathrm{Tb}^{*}$ & 0.90 & 0.07 & 0.09 & 0.19 & 0.05 & 0.04 & 0.06 & 0.03 & 0.09 & 0.00 & 0.02 & 0.14 & 0.07 & 0.01 & 0.08 & 0.23 & 0.11 & 0.15 & 0.07 \\
\hline Dy* & 4.52 & 0.30 & 0.35 & 1.72 & 0.25 & 0.50 & 0.40 & 0.18 & 0.43 & 0.35 & 0.13 & 0.73 & 0.26 & 0.14 & 0.70 & 1.18 & 0.32 & 1.33 & 0.67 \\
\hline $\mathrm{Yb}^{*}$ & 1.80 & 0.14 & 0.46 & 0.33 & 0.11 & 0.08 & 0.20 & 0.14 & 0.30 & 0.09 & 0.07 & 0.50 & 0.20 & 0.05 & 0.12 & 0.58 & 0.16 & 0.24 & 0.12 \\
\hline $\mathrm{Hf}^{*}$ & 0.53 & 0.25 & 0.60 & 0.76 & 0.25 & 0.20 & 0.35 & 0.18 & 0.70 & 0.17 & 0.04 & 0.60 & 0.40 & 0.16 & 0.26 & 0.87 & 0.18 & 0.14 & 0.36 \\
\hline $\mathrm{Ta}^{*}$ & 0.21 & 0.09 & 0.27 & 0.13 & 0.09 & 0.05 & 0.14 & 0.04 & 0.18 & 0.07 & 0.02 & 0.22 & 0.13 & 0.02 & 0.08 & 0.12 & 0.10 & 0.03 & 0.07 \\
\hline $\mathrm{Pb}^{* *}$ & 5.42 & 6.24 & 7.74 & 5.32 & 3.22 & 3.06 & 5.28 & 8.20 & 6.80 & 3.70 & 2.14 & 18.32 & 3.60 & 0.82 & 3.45 & 2.68 & 18.42 & 3.18 & 27.14 \\
\hline
\end{tabular}




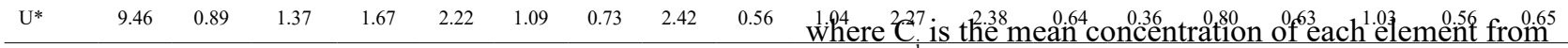

* - determined by NAA; ** - determined by TXRF

The mean concentration of the elements in the moss samples from Dalat decreased as: $\mathrm{K}>\mathrm{Fe}>\mathrm{Si}>\mathrm{Al}>\mathrm{S}>$ $\mathrm{P}>\mathrm{Cl}>\mathrm{Zn}>\mathrm{Na}>\mathrm{Mg}>\mathrm{Ti}>\mathrm{Mn}>\mathrm{Rb}>\mathrm{Cu}>\mathrm{V}>\mathrm{Ce}$ $>\mathrm{Ba}>\mathrm{Cr}>\mathrm{Pb}>\mathrm{La}>\mathrm{I}>\mathrm{Y}>\mathrm{Br}>\mathrm{As}>\mathrm{Ni}>\mathrm{U}>\mathrm{Th}>$ $\mathrm{Co}>\mathrm{Sm}>\mathrm{Sc}>\mathrm{Dy}>\mathrm{Cs}>\mathrm{Hf}>\mathrm{Yb}>\mathrm{Kr}>\mathrm{Sb}>\mathrm{Se}>\mathrm{Eu}$ $>\mathrm{Tb}>\mathrm{Ta}$.

The contamination factor $(\mathrm{CF})$ is the ratio of the mean concentration of each heayy metal to the average of the three lowest concentrations $\overline{\bar{d}}$ the same metal. CF was calculated from (1) (Hakanson 1980; Zhou et al. 2017): the investigated area; and $\mathrm{BG}_{\mathrm{i}}$ is the average value of the three sample sites with the lowest concentration of the corresponding metal from the investigated area.

According to Fernández and Carballeira (2001), the contamination factors $(\mathrm{CF})$ can be used to determine the contamination levels of each element in the sample. The $\mathrm{CF}$ values comprise of six categories: $\mathrm{CF}<1$ (category I): no contamination, $1<\mathrm{CF} \leq 2$ (category II): suspected contamination, $2<\mathrm{CF} \leq 3.5$ (category III): slight contamination, $3.5<\mathrm{CF} \leq 8$ : moderate contamination (category IV), $8<\mathrm{CF} \leq 27$ : serious contamination

(category V), and CF $>27$ : extreme contamination (category VI). Contamination factors are shown in Table 3. It can be seen from Table 3 that $\mathrm{Tb}$ air pollution $(\mathrm{CF}=11.9)$ is very serious (category VI).

TABLE 3. The contamination factors

\begin{tabular}{lllllllllllllllllllll}
\hline Element & $\mathrm{Na}$ & $\mathrm{Mg}$ & $\mathrm{Al}$ & $\mathrm{Si}$ & $\mathrm{P}$ & $\mathrm{S}$ & $\mathrm{Cl}$ & $\mathrm{K}$ & $\mathrm{Sc}$ & $\mathrm{Ti}$ & $\mathrm{V}$ & $\mathrm{Cr}$ & $\mathrm{Mn}$ & $\mathrm{Fe}$ & $\mathrm{Co}$ & $\mathrm{Ni}$ & $\mathrm{Cu}$ & $\mathrm{Zn}$ & $\mathrm{As}$ & $\mathrm{Se}$ \\
\hline $\mathrm{CF}$ & 2.0 & 1.4 & 3.0 & 1.6 & 1.8 & 1.6 & 3.9 & 2.8 & 5.1 & 3.8 & 1.9 & 2.9 & 1.8 & 4.4 & 3.9 & 3.1 & 1.7 & 4.0 & 5.0 & 1.8 \\
Category & $\mathrm{II}$ & II & III & II & II & II & III & III & IV & IV & II & III & II & IV & IV & III & II & IV & IV & II \\
\hline El & $\mathrm{Br}$ & $\mathrm{Kr}$ & $\mathrm{Rb}$ & $\mathrm{Y}$ & $\mathrm{Sb}$ & $\mathrm{I}$ & $\mathrm{Cs}$ & $\mathrm{Ba}$ & $\mathrm{La}$ & $\mathrm{Ce}$ & $\mathrm{Sm}$ & $\mathrm{Eu}$ & $\mathrm{Tb}$ & $\mathrm{Dy}$ & $\mathrm{Yb}$ & $\mathrm{Hf}$ & $\mathrm{Ta}$ & $\mathrm{Pb}$ & $\mathrm{Th}$ & $\mathrm{U}$ \\
\hline
\end{tabular}

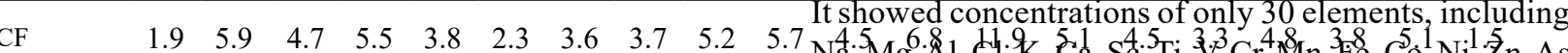

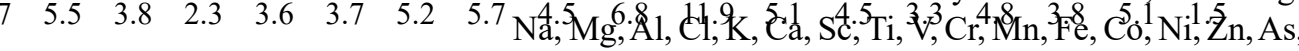

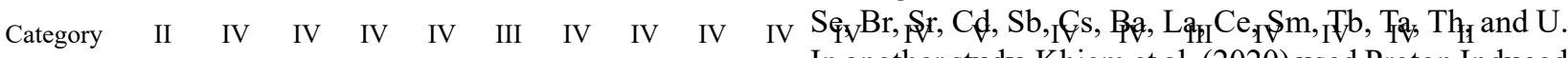

A comparison of these results with those obtained by previous investigations of Barbula indica moss in Vietnam and other mosses in European countries (Barandovski et al. 2013) was carried out. The results of the comparison are listed in Table 4. Ho Chi Minh City (in the south) and Hanoi (in the north) are the biggest city and the capital of Vietnam, respectively. They have many companies and factories. Hue and Hoi An are cities in the middle of Vietnam that have many industrial parks. Doan Phan et al. (2019) used the INAA technique for analysis. In another study, Khiem et al. (2020) used Proton Induced $\mathrm{X}$-ray Emission to detect trace elements in Barbula indica moss from Hanoi; it showed the concentration of 22 elements: $\mathrm{Mg}, \mathrm{Al}, \mathrm{Si}, \mathrm{P}, \mathrm{S}, \mathrm{Cl}, \mathrm{K}, \mathrm{Ca}, \mathrm{Ti}, \mathrm{Cr}, \mathrm{Mn}, \mathrm{Fe}$, $\mathrm{Ni}, \mathrm{Cu}, \mathrm{Zn}, \mathrm{As}, \mathrm{Br}, \mathrm{Rb}, \mathrm{Sr}, \mathrm{Zr}, \mathrm{Ba}$, and $\mathrm{Pb}$.

For Hue, Hoi An, and Ho Chi Minh City, the element concentrations are higher than in our work. For Hanoi, all element concentrations are higher than in Dalat. The element concentrations of $\mathrm{Mg}, \mathrm{Al}, \mathrm{Si}, \mathrm{S}, \mathrm{Cl}, \mathrm{K}, \mathrm{Ti}, \mathrm{Cr}$, $\mathrm{Mn}, \mathrm{Fe}, \mathrm{Cu}, \mathrm{As}, \mathrm{Br}, \mathrm{Rb}, \mathrm{Ba}$, and $\mathrm{Pb}$ in Hanoi are higher than in Dalat by 17.2, 4.2, 8.9, 2.7, 3.7, 2.4, 5.5, 3.5, 2.3, $2.5,2.3,5.5,10.5,12.3,132$, and 32.7 times, respectively. 
Concentrations of barium and lead in Hanoi are especially high compared to Dalat.

TABLE 4. Mean element concentrations due to atmospheric deposition from Barbula indica mosses in Dalat and other cities in Vietnam and other mosses in Europe (in mg. $\mathrm{kg}^{-1}$ )

\begin{tabular}{|c|c|c|c|c|c|c|}
\hline \multirow{2}{*}{ Element } & \multirow{2}{*}{$\begin{array}{c}\text { Dalat } \\
\text { (our work) }\end{array}$} & \multicolumn{3}{|c|}{ Doan Phan et al. 2019} & \multirow{2}{*}{$\begin{array}{c}\text { Khiem et al. } \\
2020 \\
\text { Hanoi } \\
\text { Capital }\end{array}$} & \multirow{2}{*}{$\begin{array}{c}\text { Barandovski et al. } \\
2013 \\
\text { Macedonia }\end{array}$} \\
\hline & & Hue & Hoi An & $\begin{array}{l}\text { Ho Chi Minh } \\
\text { City }\end{array}$ & & \\
\hline $\mathrm{Na}$ & 200 & 620 & 1310 & 930 & & \\
\hline $\mathrm{Mg}$ & 225 & 1550 & 1620 & 1290 & 3866.66 & 1900 \\
\hline $\mathrm{Al}$ & 2395 & 5800 & 3200 & 4800 & 10591.19 & 1900 \\
\hline $\mathrm{Si}$ & 4446 & & & & 39595.76 & \\
\hline $\mathrm{P}$ & 840 & & & & 1100.14 & 1100 \\
\hline $\mathrm{S}$ & 1184 & & & & 3238.92 & \\
\hline $\mathrm{Cl}$ & 469 & 1700 & 2100 & 780 & 1711.59 & \\
\hline K & 5932 & 16000 & 17000 & 12000 & 14401.56 & 4600 \\
\hline $\mathrm{Sc}$ & 0.98 & 1.09 & 0.80 & 1.81 & & \\
\hline $\mathrm{Ti}$ & 126 & 271 & 205 & 524 & 691.60 & \\
\hline $\mathrm{V}$ & 9.64 & 12.20 & 5.11 & 8.08 & & 3.5 \\
\hline $\mathrm{Cr}$ & 7.14 & 11 & 6.80 & 19.90 & 26.73 & 3.5 \\
\hline $\mathrm{Mn}$ & 76 & 74 & 88 & 77 & 170.95 & 130 \\
\hline $\mathrm{Fe}$ & 2408 & 3720 & 4810 & 5430 & 6025.18 & 1500 \\
\hline Co & 1.04 & 1.40 & 1.01 & 3.28 & & \\
\hline $\mathrm{Ni}$ & 2.46 & 4.20 & 2.70 & 9.50 & 4.42 & 3.5 \\
\hline $\mathrm{Cu}$ & 12.01 & & & & 27.20 & 3.5 \\
\hline $\mathrm{Zn}$ & 245 & 126 & 254 & 178 & 397.53 & 20 \\
\hline As & 2.93 & 2.30 & 3.00 & 4.10 & 16.11 & \\
\hline $\mathrm{Se}$ & 0.18 & 1.40 & 0.70 & 0.39 & & \\
\hline $\mathrm{Br}$ & 3.45 & 10.40 & 7.70 & 12.80 & 36.11 & \\
\hline $\mathrm{Kr}$ & 1.18 & & & & & \\
\hline $\mathrm{Rb}$ & 13.42 & & & & 151.17 & \\
\hline $\mathrm{Y}$ & 4.21 & & & & & \\
\hline $\mathrm{Sb}$ & 0.23 & 1.40 & 0.89 & 1.00 & & \\
\hline I & 4.69 & & & & & \\
\hline Cs & 0.62 & 1.58 & 1.18 & 5.30 & & \\
\hline $\mathrm{Ba}$ & 11.68 & 58 & 83 & 101 & 1545.55 & 34 \\
\hline $\mathrm{La}$ & 5.73 & 3.10 & 2.24 & 5.60 & & \\
\hline $\mathrm{Ce}$ & 8.82 & 6.20 & 4.20 & 11.70 & & \\
\hline $\mathrm{Sm}$ & 0.99 & 0.51 & 0.35 & 1.15 & & \\
\hline $\mathrm{Eu}$ & 0.14 & & & & & \\
\hline $\mathrm{Tb}$ & 0.13 & & & & & \\
\hline Dy & 0.76 & & & & & \\
\hline $\mathrm{Yb}$ & 0.30 & & & & & \\
\hline $\mathrm{Hf}$ & 0.37 & & & & & \\
\hline $\mathrm{Ta}$ & 0.11 & 0.09 & 0.07 & 0.15 & & \\
\hline $\mathrm{Pb}$ & 7.09 & & & & 231.55 & 4.6 \\
\hline
\end{tabular}




\begin{tabular}{cccc} 
Th & 1.15 & 1.78 & 0.86 \\
$\mathrm{U}$ & 1.62 & 0.62 & 0.23 \\
\hline \multicolumn{3}{c}{ DATA PROCESSING AND STATISTICAL ANALYSIS }
\end{tabular}

Factor analysis is commonly used for statistical analysis in environmental science. Data processing was performed using the Statistica- 8 software. The relationship of each variable to the underlying factor is expressed though the factor logding, which ranges from -1.0 to 1.0. A factor loading close to 1 indicates a strong effect on the element concentration.

In our multivariate statistical analysis, eleven elements of interest to us were selected; namely, V, As, $\mathrm{Fe}, \mathrm{Zn}, \mathrm{Se}, \mathrm{Rb}, \mathrm{Sb}, \mathrm{Cs}, \mathrm{Al}, \mathrm{Cu}$, and $\mathrm{Pb}$. The correlation coefficients of the selected elements in the moss samples

of Dalat are presented in Table 5. Four factor loadings, F1, F2, F3, and F4, can explain more than $86 \%$ of the variance.

TABLE 5. Eigen values for the factors

\begin{tabular}{lcccc}
\hline $\begin{array}{l}\text { Factor } \\
\text { loading }\end{array}$ & $\begin{array}{l}\text { Eigen } \\
\text { value }\end{array}$ & Cumulative & Variability (\%) & Cumulative (\%) \\
\hline F1 & 5.07 & 5.07 & 42.28 & 42.28 \\
F2 & 2.50 & 7.58 & 20.87 & 63.16 \\
F3 & 1.63 & 9.21 & 13.61 & 76.76
\end{tabular}

F4

1.16

10.37

9.63

The factor loadings and factor scores are shown in Tables 6 and 7, respectively. Earlier studies by Cucu-Man et al. (2004) and Pacyna and Pacyna (2001) have used factor score and factor loading to explain the results of moss biomonitoring studies. Factor loadings after Varimax normalized rotation and explained variance are shown in Table 6. Factor scores used to estimate factor portions

at the sites are presented in Table 7 . The main pollution sources in our work can be explained in the next part.

TABLE 6. Matrix of rotated factor loadings (11 selected elements)

\begin{tabular}{lcccc}
\hline Element & F1 & F2 & F3 & F4 \\
\hline V & -0.013 & 0.016 & $\mathbf{0 . 9 2 1}$ & -0.022 \\
$\mathrm{As}$ & $\mathbf{0 . 8 3 6}$ & 0.116 & -0.166 & -0.101 \\
$\mathrm{Fe}$ & $\mathbf{0 . 8 6 5}$ & 0.242 & 0.259 & 0.017 \\
$\mathrm{Zn}$ & -0.185 & $\mathbf{0 . 7 3 2}$ & -0.321 & 0.413 \\
$\mathrm{Se}$ & $\mathbf{0 . 8 3 1}$ & -0.173 & 0.411 & 0.061 \\
$\mathrm{Rb}$ & $\mathbf{0 . 6 9 2}$ & $\mathbf{0 . 5 9 9}$ & -0.304 & -0.096 \\
$\mathrm{Sb}$ & 0.056 & 0.090 & 0.022 & $\mathbf{0 . 9 6 4}$ \\
$\mathrm{Cs}$ & $\mathbf{0 . 9 4 3}$ & -0.041 & 0.018 & 0.023 \\
$\mathrm{Al}$ & 0.456 & 0.221 & $\mathbf{0 . 6 2 3}$ & 0.045
\end{tabular}




\begin{tabular}{lllll}
$\mathrm{Cu}$ & 0.244 & $\mathbf{0 . 9 1 6}$ & 0.128 & 0.224 \\
$\mathrm{~Pb}$ & -0.021 & $\mathbf{0 . 8 5 9}$ & 0.299 & -0.211 \\
\hline
\end{tabular}

TABLE 7. Factor scores for each sampling site

\begin{tabular}{lcccc}
\hline $\begin{array}{l}\text { Sampling } \\
\text { Site }\end{array}$ & F1 & F2 & F3 & F4 \\
\hline DL01 & $\mathbf{2 . 5 3}$ & -0.41 & -0.7 & 0.52 \\
DL02 & -0.22 & 0.009 & 0.15 & -0.63 \\
DL03 & $\mathbf{0 . 9 9}$ & 0.46 & 0.41 & $\mathbf{1 . 0 2}$ \\
DL04 & $\mathbf{1 . 0 1}$ & -0.87 & -0.13 & -0.86 \\
DL05 & -0.15 & -0.25 & -0.64 & -0.277 \\
DL06 & -0.78 & -0.56 & -0.14 & -0.41 \\
DL07 & $\mathbf{0 . 7 2}$ & -0.07 & -0.21 & -0.59 \\
DL08 & -0.69 & $\mathbf{1 . 6 4}$ & -1.58 & $\mathbf{1 . 7 6}$ \\
DL09 & $\mathbf{1 . 6 6}$ & 0.37 & 0.42 & -0.81 \\
DL10 & -0.33 & -0.54 & 0.17 & -0.65 \\
DL11 & -1.02 & -0.85 & -0.7 & 0.67 \\
DL12 & 0.56 & $\mathbf{1 . 6 1}$ & -0.64 & -0.84 \\
DL13 & -0.46 & -0.64 & -0.02 & 0.32 \\
DL14 & -1.05 & -0.92 & -1.02 & 0.001 \\
DL15 & -0.5 & -0.97 & $\mathbf{1 . 5 4}$ & -0.32 \\
DL16 & 0.16 & -0.82 & $\mathbf{1 . 7 9}$ & $\mathbf{2 . 7 1}$ \\
DL17 & -0.63 & $\mathbf{2}$ & -0.15 & 0.32
\end{tabular}

\begin{tabular}{lllll} 
DL18 & -0.88 & -0.74 & -0.9 & -0.92 \\
DL19 & -0.89 & $\mathbf{1 . 5 8}$ & $\mathbf{2 . 3 8}$ & -0.99 \\
\hline
\end{tabular}

Factor 1, the strongest, explains $42.28 \%$ of the total variance. It is heavily loaded by Cs (0.943), As (0.836), $\mathrm{Fe}(0.865)$, Se (0.831), and Rb (0.692). These elements are found in the Earth's crust, and Fe, especially, is the fourth most abundant element in the Earth's crust. Arsenic is a naturally occurring element that is widely distributed in soils and minerals. In addition, Dalat is a major vegetable-growing region in Vietnam. Farmers use a variety of pesticides that contain As and Se to grow vegetables, so Dalat's soil may be highly polluted by As and Se. The consequence is that it is easy to find As and Se in the dust of Dalat. Therefore, Factor- 1 is most likely from windblown dust. The highest F1 scores were found at sites: DL01(2.53), DL03 (0.99), DL07 (0.72), DL09 (1.66), and DL13 (1.01), which are within $2 \mathrm{~km}$ of a national highway and surrounded by vegetable farms.

Factor 2 is smaller than $\mathrm{F} 1$ and is the second strongest. It represents $20.87 \%$ of the total variance. It effects the factor loadings of $\mathrm{Zn}$ (0.732), $\mathrm{Rb}$ (0.599), $\mathrm{Cu}$ (0.916), and $\mathrm{Pb}(0.859)$. Zinc, $\mathrm{Cu}$, and $\mathrm{Pb}$ are well known to be in emissions from brake and tire wear (Adamiec 2017; Nguyen et al. 2018). The highest F2 scores were found at sites DL08 (1.64), DL12 (1.61), DL17 (2.00), and DL19 (1.58). These sites are near a national highway and other roads. This suggests the pollution source related to the F2 factor is the traffic emissions of gasoline-burning vehicles, especially cars and motorbikes.

Factor 3 is $13.61 \%$ of the total variance. It is heavily 
loaded by V (0.921) and to a lesser extent by $\mathrm{Al}(0.623)$. It is confirmed that $\mathrm{V}$ is emitted from the combustion of fossil fuels and oil (Kousehlar \& Windom 2019; Visschedijk et al. 2013). In Dalat, the use of fossil fuels for cooking and people's daily activities is still common. Furthermore, farmers in Dalat regularly use diesel-powered engines to irrigate coffee and other agricultural crops. These activities can be the main sources of vanadium emissions into the atmosphere in Dalat. The highest Factor 3 scores were found at sites: DL16 (1.79), DL15 (1.54), and DL19 (2.38), where there are flower and rice farms. Therefore, it may be suggested that Factor 3 is related to the combustion of fossil fuels and oil.

Factor 4 represents $9.63 \%$ of the total variance. It contains $\mathrm{Sb}$ for which the factor loading is 0.964 . The highest Factor 4 scores were found at sites: DL03 (1.02), DL08 (1.76), DL16 (2.71). The Sb concentration is very low at all sites (maximum value is $0.64 \mathrm{ppm}$ ). Element $\mathrm{Sb}$ originates mainly from mining, especially open pit gold-antimony mines. DL03 and DL16 are located at the foothills of Elephant Mountain which has antimony ore, and DL08 is at a local antimony mining area (Hon Bo Mountain) where there was an antimony factory in 1990s. Therefore, Factor 4 should be related to antimony mining in Dalat.

\section{CONCLUSION}

For the first time, the well-known moss biomonitoring method has been applied to investigate the pollution of chemical elements in the air of Dalat, a large tourist city in Vietnam. Barbula indica moss samples were collected at 19 different locations in Dalat. By combining both NAA and TXRF analysis methods, the concentration of 40 chemical elements, including $\mathrm{Na}, \mathrm{Mg}, \mathrm{Al}, \mathrm{Si}, \mathrm{P}, \mathrm{S}, \mathrm{Cl}$, $\mathrm{K}, \mathrm{Sc}, \mathrm{Ti}, \mathrm{V}, \mathrm{Cr}, \mathrm{Mn}, \mathrm{Fe}, \mathrm{Co}, \mathrm{Ni}, \mathrm{Cu}, \mathrm{Zn}, \mathrm{As}, \mathrm{Se}, \mathrm{Br}, \mathrm{Kr}$, Rb, Y, Sb, I, Cs, Ba, La, Ce, Sm, Eu, Tb, Dy, Yb, Hf, Ta, $\mathrm{Pb}, \mathrm{Th}$, and $\mathrm{U}$ were determined in the moss samples. A comparison of the concentrations of elements in the air of Dalat with other cities that we have investigated by the same method shows that the level of trace element air pollution in Dalat is lower. Contamination factors have been estimated using the concentration data. The results show that Dalat's air is suspected of being polluted by $\mathrm{Na}, \mathrm{Mg}, \mathrm{Si}, \mathrm{P}, \mathrm{S}, \mathrm{V}, \mathrm{Mn}, \mathrm{Cu}, \mathrm{Se}, \mathrm{Br}$, and U; slightly polluted by $\mathrm{Al}, \mathrm{Cl}, \mathrm{Cr}, \mathrm{Ni}, \mathrm{S}$, and $\mathrm{Hf}$; moderately polluted by $\mathrm{Sc}, \mathrm{Fe}, \mathrm{Zn}, \mathrm{As}, \mathrm{Kr}, \mathrm{Rb}, \mathrm{Y}, \mathrm{Sb}, \mathrm{Cs}, \mathrm{Ba}, \mathrm{La}, \mathrm{Ce}, \mathrm{Sm}, \mathrm{Eu}$, $\mathrm{Dy}, \mathrm{Yb}, \mathrm{Ta}, \mathrm{Pb}$, and $\mathrm{Th}$; and seriously polluted by $\mathrm{Tb}$. Factor analysis has been applied to the concentrations of 11 elements, including $\mathrm{V}, \mathrm{As}, \mathrm{Fe}, \mathrm{Zn}, \mathrm{Se}, \mathrm{Rb}, \mathrm{Sb}, \mathrm{Cs}, \mathrm{Al}, \mathrm{Cu}$, and $\mathrm{Pb}$ to find the possible sources. The analysis shows that windblown dust, emissions from vehicles, the use of pesticides, the combustion of fossil fuels, and antimony mining are the main sources of air pollution in Dalat.

\section{ACKNOWLEDGEMENTS}

This work is supported by the Ministry of Education and Training of Vietnam under the project B2019-DLA-04 and partly by the International Centre of Physics at the Institute of Physics (Grant ICP.2021.11). We acknowledge the critical comments from anonymous reviewers and editors.

\section{REFERENCES}

Abdullah, M.Z., Saat, A. \& Hamzah, Z. 2011. Assessment of the impact of petroleum and petrochemical industries to the surrounding areas in Malaysia using mosses as bioindicator supported by multivariate analysis. Environmental Monitoring and Assessment 184(6): 3959-3969.

Adamiec, E. 2017. Chemical fractionation and mobility of traffic-related elements in road environments. Environmental Geochemistry and Health 39: 1457-1468.

Barandovski, L., Stafilov, T., Sajn, R., Frontasyeva, M. \& Baceva, K. 2013. Air pollution study in Macedonia using a moss biomonitoring technique, ICP-AES and AAS. Macedonian Journal of Chemistry and Chemical Engineering 32(1): 89-107.

Cucu-Man, S., Mocanu, R., Culicov, O., Steinnes, E. \& Frontasyeva, M. 2004. Atmospheric deposition of metals in Romania studied by biomonitoring using the epiphytic moss Hypnum cupressiforme. International Journal of Environmental Analytical Chemistry 84(11): 845-854.

Doan Phan, T.T., Trinh, T.T., Khiem, L.H., Frontasyeva, M. \& Quyet, N.H. 2019. Study of airborne trace element pollution in central and southern Vietnam using moss (Barbula indica) technique and neutron activation analysis. Asia-Pacific Journal of Atmospheric Sciences 55: 247253.

Fernández, J. \& Carballeira, A. 2001. A comparison of indigenous mosses and top soils for use in monitoring atmospheric heavy metal deposition in Galicia (northwest Spain). Environmental Pollution 114(3): 431-441.

Fernández, J. \& Carballeira, A. 2000. Differences in the responses of native and transplanted mosses to atmospheric pollution: A possible role of selenium. Environmental Pollution 110(1): 73-78.

Frontasyeva, M. \& Harmens, H. 2020. Monitoring of Atmospheric Deposition of Heavy Metals, Nitrogen and POPs in Europe using Bryophytes: 2020 survey. ICP Vegetation. United Kingdom: United Nations Economic Commission for Europe Convention on Long-Range Transboundary Air Pollution.

Frontasyeva, M., Galinskaya, T.Y., Krmar, M., Matavuly, M., Pavlov, S.S., Povtoreyko, E.A., Radnovic, D. \& Steinnes, 
E. 2004. Atmospheric deposition of heavy metals in northern Serbia and Bosnia-Herzegovina studied by the moss biomonitoring, neutron activation analysis and GIS technology. Journal of Radioanalytical and Nuclear Chemistry 259(1): 141-144.

Gjengedal, E. \& Steinnes, E. 1990. Uptake of metal ions in moss from artificial precipitation. Environmental Monitoring and Assessment 14(1): 77-87.

Hakanson, L. 1980. An ecological risk index for aquatic pollution control: A sedimentological approach. Water Research 14(8): 975-1001.

Harmens, H., Norris, D.A., Steinnes, E., Kubin, E., Piispanen, J., Alber, R., Aleksiayenak, Y., Blum, O., Coşkun, M., Dam, M., De Temmerman, L., Fernández, J.A., Frolova, M., Frontasyeva, M., González-Miqueo, L., Grodzińska, K., Jeran, Z., Korzekwa, S., Krmar, M., Kvietkus, K., Leblond, S., Liiv, S., Magnússon, S.H., Maňkovská, B., Pesch, R., Rühling, Å., Santamaria, J.M., Schröder, W., Spiric, Z., Suchara, I., Thöni, L., Urumov, V., Yurukova, L. \& Zechmeister, H.G. 2010. Mosses as biomonitors of atmospheric heavy metal deposition: Spatial patterns and temporal trends in Europe. Environmental Pollution 158(10): 3144-3156.

Ho, M.D. \& Pham, D.H. 2003. The application and development of $\mathrm{k} 0$-standardization method of neutron activation analysis at Dalat research reactor. Journal of Radioanalytical and Nuclear Chemistry 257: 643-647.

Ho, M.D. Tran, Q.T., Ho, V.D. \& Nguyen, T.S. 2016 a. Determination of multielement composition of Vietnamese marine sediment and tuna fish by k0-NAA. Journal of Radioanalytical and Nuclear Chemistry 309(1): 235-241.

Ho, M.D., Tran, Q.T., Ho, V.D. \& Nguyen, T.S. 2016b. Quality evaluation of the k0-NAA at the Dalat research reactor. Journal of Radioanalytical and Nuclear Chemistry 309(1): 135-143.

Khiem, L.H., Sera, K., Hosokawa, T. \& Quyet, N.H. 2020. Assessment of atmospheric deposition of metals in Hanoi using the moss bio-monitoring technique and proton induced x-ray emission. Journal of Radioanalytical and Nuclear Chemistry 324(4): 43-54.

Kousehlar, M. \& Widom, E. 2019. Sources of metals in atmospheric particulate matter in Tehran, Iran: Tree bark biomonitoring. Applied Geochemistry 104: 71-82.

Lee, C.S.L., Li, X., Zhang, G., Peng, X. \& Zhang, L. 2005. Biomonitoring of trace metals in the atmosphere using moss (Hypnum plumaeforme) in the Nanling Mountains and the Pearl River Delta, Southern China. Atmospheric Environment 39(3): 397-407.

Munar, M.P., Oreiro, R.R.B. \& Hipol, R.L. 2014. Ectohydric moss, Thuidium tamariscellum, monitors atmospheric lead $(\mathrm{Pb})$ pollution in Baguio City, Philippines. Tropical Plant Research 1(1): 4-7.

Nguyen, T.N.T., Le, H.A., Mac, T.M.T., Nguyen, T.T.N., Pham, V.H. \& Bui, Q.H. 2018. Current status of $\mathrm{PM}_{25}$ pollution and its mitigation in Vietnam. Global Environmental Research 22: 73-83

Pacyna, J.M. \& Pacyna, E.G. 2001. An assessment of global and regional emissions of trace metals to the atmosphere from anthropogenic sources worldwide. Environmental Reviews 9(4): 269-298

Rühling, Å. \& Tyler, G. 1970. Sorption and retention of heavy metals in the woodland moss Hylocomium splendens (Hedw.) BR. et Sch. Oikos 21(1): 92-97.

Rühling, Å. \& Tyler, G. 1969. Ecology of heavy metals - a regional and historical study. Botaniska Notiser 122(1): 248-259.

Rühling, Å. \& Tyler, G. 1968. An ecological approach to the lead problem. Botaniska Notiser 121(1): 321-342.

Sucharová, J. \& Suchara, I. 1998. Atmospheric deposition levels of chosen elements in the Czech Republic determined in the framework of the international Bryomonitoring program 1995. Science of The Total Environment 223(1): $37-52$.

Towett, E.K., Shepherd, K.D. \& Cadisch, G. 2013. Quantification of total element concentrations in soils using total X-ray fluorescence spectroscopy (TXRF). Science of The Total Environment 463-464: 374-388.

Visschedijk, A.H.J., Denier van der Gon, H.A.C., Hulskotte, J.H.J. \& Quass, U. 2013. Anthropogenic Vanadium emissions to air and ambient air concentrations in NorthWest Europe. E3S Web of Conferences: Proceedings of the $16^{\text {th }}$ International Conference on Heavy Metals in the Environment 1, 03004. United Kingdom: EDP Sciences. pp. 1-4.

Zhou, X., Chen, Q., Liu, C. \& Fang, Y. 2017. Using moss to assess airborne heavy metal pollution in Taizhou, China International Journal of Environmental Research and Public Health 14(4): 430-442.

Nguyen An Son* \& Nguyen Thi Minh Sang

Department of Nuclear Engineering

Dalat University

Dalat, Lam Dong

Vietnam

\section{Le Hong Khiem}

Institute of Physics

Vietnam Academy of Science and Technology

Hanoi

Vietnam

\section{Le Hong Khiem}

Graduate University for Science and Technology

Vietnam Academy of Science and Technology

Hanoi

Vietnam

Doan Phan Thao Tien

Nhatrang Institute of Technology Research and Application

02 Hungvuong Street

Vietnam Academy of Science and Technology

Nhatrang City

Vietnam

Ho Huu Thang

Dalat Nuclear Research Institute

01 Nguyen Tu Luc

Dalat, Lam Dong

Vietnam

*Corresponding author; email: sonna@dlu.edu.vn 